\title{
Androgenic TRPM8 activity drives sexual dimorphism in a murine model of chronic migraine
}

\section{David Alarcón-Alarcón}

Universidad Miguel Hernández

David Cabañero ( $\square$ dcabanero@umh.es )

Universidad Miguel Hernández https://orcid.org/0000-0002-1133-0908

Jorge de Andrés-López

Universidad Miguel Hernández

Gregorio Fernández-Ballester

Universidad Miguel Hernandez

Asia Fernández-Carvajal

University Miguel Hernández

Antonio Ferrer-Montiel

Instituto de Biología Molecular y Celular

\section{Article}

Keywords: Chronic migraine, sexual dimorphism, TRPM8, testosterone, TRPA1, nitroglycerin, pain resilience

Posted Date: October 19th, 2021

DOl: https://doi.org/10.21203/rs.3.rs-960579/v1

License: (1) (1) This work is licensed under a Creative Commons Attribution 4.0 International License.

Read Full License

Version of Record: A version of this preprint was published at Nature Communications on October 22nd, 2022. See the published version at https://doi.org/10.1038/s41467-022-33835-3. 


\section{Abstract}

The mechanisms contributing to the high prevalence of chronic migraine in females are yet elusive. Here, we used a mouse model of nitroglycerin-induced chronic migraine that displays a sexual dimorphic phenotype and unveiled a role of TRPM8 as a testosterone receptor that provides antinociceptive resilience exclusively in males. Nitroglycerin induced similar mechanosensitivity to both sexes trough activation of TRPA1 channels, but triggered persistent hypersensitivity solely in females, as males readily recovered from the migraine crisis. Notably, we found that testosterone exerted an antinociceptive activity through its interaction with the TRPM8 channel. Downregulation of this protective mechanism in males led to persistent mechanical hypersensitivity, whereas administration of testosterone to females favoured their recovery. Thus, our findings reveal a novel protective function of TRPM8 through pre-clinical models of acute and chronic pain and highlights the interest of molecular solutions mimicking the pain-relieving activity of testosterone on TRPM8.

\section{Introduction}

Chronic migraine is a highly prevalent and recurrent headache affliction particularly severe in women ${ }^{1}$. The mechanisms underlying such sex dimorphism of chronic migraine remain largely unknown. A migraine model with high predictive validity is the sensory sensitization induced by nitroglycerin (NTG) ${ }^{2}$. Acute NTG treatments lead to a delayed hypersensitivity to mechanical stimulation that lasts hours in humans and rodents ${ }^{2,3}$. Furthermore, repeated NTG exposure causes a chronic hypersensitivity that lasts several weeks in murine models ${ }^{4}$. This chronic hypersensitivity is characterized by generalized cutaneous sensitization, which has also been described as a reliable predictor of migraine chronification in humans ${ }^{5}$.

Multiple migraine triggers including NTG have demonstrated a crucial involvement of transient receptor potential ankyrin 1 (TRPA1) in rodent models of acute migraine ${ }^{3,6}$. Indeed, TRPA1 is expressed in primary afferent neurons innervating the meninges where its activation favours the release of a-calcitonin generelated peptide (aCGRP) ${ }^{6-8}$ a neuropeptide that plays a pivotal role in migraine development ${ }^{2,9,10}$. An additional TRP tightly associated with the expression of chronic migraine in humans is the transient receptor potential melastatin 8 (TRPM8). Several single-nucleotide polymorphisms affecting TRPM8 have been linked to migraine ${ }^{11,12}$ and TRPM8 agonists such as menthol have been used medicinally for the alleviation of migraine-related pain ${ }^{13,14}$.

TRPM8 is a cation channel expressed in primary afferent neurons, known for being the menthol receptor and the principal detector of environmental cold ${ }^{15-18}$. As such, TRPM8 activity shows modulatory effects on thermal and mechanical hypersensitivity in preclinical models of pain ${ }^{19}$. However, its presence in internal structures kept at euthermic temperature ${ }^{20}$ and its recent description in central brain areas ${ }^{21}$ suggests additional functions of this protein that may go beyond cold perception. In this line, TRPM8 was previously described as a testosterone receptor in cellular models ${ }^{22}$. While high testosterone levels have 
been associated with decreased pain sensitivity in mice ${ }^{23,24}$ and humans ${ }^{25}$, it is unknown whether testosterone-TRPM8 interactions could have functional relevance in pain perception.

Here we implemented a murine model of chronic migraine that displays a sexual dimorphism characterized by enhanced pain sensitivity of females as described in humans. Mechanical sensitivity was assessed in mice of both sexes chronically exposed to NTG and the participation of TRPA1 and TRPM8 was evaluated through genetic and pharmacological approaches. To dissect the functional and molecular consequences of TRPA1 and TRPM 8 activities, murine cultures of trigeminal neurons and transfected cell lines expressing murine and human receptors were evaluated. After finding a malespecific function of TRPM8, the role of testosterone-TRPM8 interactions and the effects of exogenous TRPM8 stimulation were elucidated in in vitro and in vivo models of acute and chronic pain. Molecular docking in murine and human ligand-receptor models provided further insight on TRPM8 function as a testosterone receptor. Collectively, our data suggest that testosterone, through its interaction with TRPM8, drives sexual dimorphism in chronic migraine and likely in other pain-related behaviours.

\section{Methods}

2.1. Animals. Adult male and female mice with a C57BL/6J background (Envigo, Horst, The Netherlands), wild-type or defective in $T r p a 7^{26}$ or Trpm $8^{17}$ were bred in the animal facility at Universidad Miguel Hernández (UMH, Elche, Alicante, Spain). TRPM8 knockout mice were a gift from Dr. F. Viana (Instituto de Neurociencias de Alicante, Alicante, Spain). Care was taken to minimize the number of animals used and the pain and stress they experienced. All experimental procedures were approved by the Animal Care and Use Committees of Universidad Miguel Hernández and the regional government and were conducted according to the ethical principles of the International Association for the Study of Pain (IASP) for the evaluation of pain in conscious animals ${ }^{27}$, the European Parliament and the Council Directive (2010/63/EU) and the Spanish law (RD 53/2013). Housing conditions were maintained at $21 \pm 1^{\circ} \mathrm{C}$ and $55 \pm 15 \%$ relative humidity in a controlled light/dark cycle (light on between 8:00 a.m. and 8:00 p.m.). Animals had free access to food and water except during manipulations and behavioural assessment. Experiments were performed blinded for NTG, genotype or pharmacological treatment depending on the studied condition.

2.2. Drugs for behavioural studies. Two NTG formulations were used: $5 \mathrm{mg} / 1.5 \mathrm{ml}$ ampoules and 50 $\mathrm{mg} / 50 \mathrm{ml}$ vials (Bioindustria LIM, Novi Liguri, Italy). The ampoules contained NTG dissolved on a vehicle made of $1 \mathrm{ml}$ propylene glycol and $0.5 \mathrm{ml}$ ethanol (Bioindustria LIM). This initial solution was dissolved in saline to obtain $1 \mathrm{mg} / \mathrm{ml} \mathrm{NTG}$, reaching final concentrations of $10 \%$ ethanol and $20 \%$ propylene glycol. The $50 \mathrm{mg} / 50 \mathrm{ml} \mathrm{NTG}$ vials contained a vehicle made of $5 \%$ dextrose and $0.105 \%$ propylene glycol in pure water (Bioindustria LIM). This NTG or its vehicle was administered without further dilution. The TRPM8 selective blocker AMTB hydrochloride (AMTB, N-(3-aminopropyl)-2-\{[(3-methylphenyl) methyl] oxy\}-N-(2-thienylmethyl) benzamide hydrochloride, Tocris, Bristol, UK) was dissolved in dimethyl sulfoxide (DMSO, Merck, Darmstadt, Germany) and was further diluted in saline to reach 2.5\% DMSO. The potent and selective TRPM8 agonist WS12 ((1R,2S,5R)-2-Isopropyl-N-(4-methoxyphenyl)-5- 
methylcyclohexanecarboxamide, Tocris) was dissolved in DMSO and diluted in corn oil to reach $2.5 \%$ DMSO. In a previous preparation, WS12 was dissolved in ethanol and diluted in 45\% 2-Hydroxypropyl- $\beta$ cyclodextrin in water to reach $5 \%$ ethanol, although precipitation was found at these concentrations. All these compounds and vehicles were injected intraperitoneally at a volume of $10 \mathrm{ml} / \mathrm{kg}$. In the formalin test, WS-12 was dissolved in DMSO and diluted in saline up to $0.6 \%$ DMSO to achieve an amount of 6 $\mathrm{nmol}$ in $20 \mu \mathrm{l}$ as previously described ${ }^{28}$. We used WS12 and not menthol or icilin as a TRPM8 agonist to avoid unspecific signaling over TRPA $1^{29}$. Testosterone (T1500, Merck) was dissolved in $45 \% 2$ Hydroxypropyl- $\beta$-cyclodextrin in water to obtain a solution of $22 \mathrm{mg} / \mathrm{ml}$.

2.3. Model of chronic migraine. Animals were exposed to a schedule of repeated NTG injections previously used to precipitate long lasting mechanical hypersensitivity 4 . Briefly, mice were injected with $10 \mathrm{mg} / \mathrm{kg}$ NTG or its vehicle every other day for 8 days (5 i.p. injections total). Mechanical sensitivity was first assessed before the repeated NTG treatment (days -1 and 0 ). Afterwards, mechanical thresholds were measured again every day of the treatment before and $2 \mathrm{~h}$ after each injection (days $0,2,4,6$ and/or 8). Then, measurements continued up to 20 days after the beginning of the procedure (days $10,12,14$, 16,18 and/or 20).

2.4. Assessment of mechanical sensitivity. Mechanical thresholds were quantified by measuring the hind paw withdrawal response to von Frey filament stimulation. Briefly, animals were placed in Plexiglas ${ }^{\circledR}$ chambers $(10 \times 10 \times 14 \mathrm{~cm})$ with a wire grid bottom through which the von Frey filaments (bending force range from 0.008 to $2 \mathrm{~g}$ ) (PanLab, Cornellá, Barcelona, Spain) were applied, by using the up-down paradigm as previously described ${ }^{30}$. The filament of $0.4 \mathrm{~g}$ was first applied. Then, the strength of the next filament was decreased when the animal responded or increased when the animal did not respond. The upper limit value $(2 \mathrm{~g})$ was recorded as a positive response even if there was no withdrawal response, and the lower limit was recorded as negative even if there was withdrawal response (0.008). This up-down procedure was stopped 4 measures after the first change in animal responding (i.e. from response to no response or from no response to response). The sequence of the last 6 responses was used to calculate the mechanical threshold. To decrease stress, prior to baseline measurements mice were habituated for 4 hours to the testing environment during 2 days. On the evaluation days, animals were also allowed to habituate for 1-2 hours before testing in order to obtain appropriate behavioural immobility. Both ipsilateral and contralateral hind paws were alternatively tested whenever possible, and stimuli were applied at a minimum of 2 min intervals to avoid hypervigilance or sensitization between successive filament applications. Filaments were completely bent before considering responses and hold up to 4-5 s to consider a negative response. Clear paw withdrawal, shaking or licking were considered as nociceptivelike responses. The responses of both hind paws were averaged to obtain the mechanical threshold of each individual.

2.5. Mouse Trigeminal Primary Cultures. Animals were sacrificed by cervical dislocation and trigeminal ganglia were extracted, micro-dissected and disaggregated mechanically and enzymatically with collagenase $48 \mu \mathrm{g} / \mathrm{ml}, 3.5 \mathrm{U} / \mathrm{mg}$ (C7657, Merck) and dispase $3 \mathrm{mg} / \mathrm{ml}, 1.79 \mathrm{U} / \mathrm{mg}$ (17105-041, Thermo Fisher Scientific, Waltham, USA). Neurons were separated from other cell types and tissue debris by 
placing the tissue homogenate over a 15\% BSA solution (073k7601, Merck) and centrifuged 7 min at 0.3 RCF. Neurons were seeded in crystals treated with Poly-L-Lysine $8.3 \mu \mathrm{g} / \mathrm{ml}$ (P9155, Merck) and laminin 5 $\mu \mathrm{g} / \mathrm{ml}$ (L2020, Merck) and incubated for $12-16 \mathrm{~h}$ at $37^{\circ} \mathrm{C}$ and $5 \% \mathrm{CO}_{2}$. Cells were kept in a hormone-free culture medium consisting of Dulbecco's Modified Eagle Medium/F12 (DMEM/F12) without Phenol Red (11039021, Thermo Fisher Scientific), MEM Vitamin Solution 1X (11120052, Thermo Fisher Scientific), Penicillin/Streptomycin 1\% (15140-022, Thermo Fisher Scientific) and home-made $\mathrm{N}_{2}$-containing insulin 4 $\mathrm{g} / \mathrm{ml}$ (12643, Merck), Putrescine hydrochloride $0.1 \mathrm{mM}$ (P7505, Merck), Sodium Selenite 3 nM (S5261 Merck), Transferrin 100 gg/mL (T2872, Molecular Probes, Eugene, USA) and NGF 25 ng/ml (G5141, Promega, Madison, USA) and hGDNF 25 ng/ml (450-10, Peprotech, London, UK).

2.6. CGRP release assay. Trigeminal cultured cells were first exposed to the exocytosis blocker DD04107 $10 \mu \mathrm{M}$ (BCN Peptides SA, San Quintí de Mediona, Spain) or its vehicle $\left(\mathrm{H}_{2} \mathrm{O}\right)$ for $1 \mathrm{~h}$. Afterwards, NTG $100 \mu \mathrm{M}$ or its vehicle (5\% dextrose and $0.105 \%$ propylene glycol) were co-applied with DD04107 $10 \mu \mathrm{M}$ or its vehicle for 30 additional min. Incubation solutions were made in culture medium and kept at $37^{\circ} \mathrm{C}$ and $5 \% \mathrm{CO}_{2}$.

2.7. Immunocytochemistry. 30 min after NTG exposure (vehicle $5 \%$ dextrose and $0.105 \%$ propylene glycol), the media was removed from the cells and the culture was washed with PBS 1X (D8662, Merck) 3 times. Afterwards, paraformaldehyde 4\% (158127, Merck) was applied for $20 \mathrm{~min}$ at room temperature. Permeabilization was achieved with Triton 100X 0.1\% v/v (P8787, Merck) for 5 min and blocking with $5 \%$ Normal Goat Serum (NGS, G9023, Merck) for $1 \mathrm{~h}$, both in PBS 1X. Neurons were labelled with rabbit antiMAP 1:250 (17490-1-AP, LabClinics, Barcelona, Spain) and mouse anti-CGRP 1:200 (AB81887, Abcam, Cambridge, UK). Secondary antibodies were Goat anti-rabbit Alexa 488 1:1000 (A11034, Thermo Fisher Scientific) and Goat anti-mouse Alexa 568 1:1000 (SAB4600400, Merck). Nuclei were stained with DAPI 1.5:10000 (D9564, Merck). Slides where mounted with mowiol (475904, Merck) and images taken with a confocal microscope (LSM 900, ZEISS, Jena, Germany). Mean fluorescence intensity for each cell was obtained, and the average value of positive cells was calculated for each picture.

2.8. Culture and transfection of human cell lines. Human embryonic kidney 293 cells (HEK293) were maintained in DMEM plus Glutamax, supplemented with 10\% Fetal Bovine Serum (FBS, Thermo Fisher Scientific) and $1 \%$ penicillin/streptomycin and incubated at $37^{\circ} \mathrm{C}$ in a $5 \% \mathrm{CO}_{2}$ atmosphere. For the NTG/Allyl Isothiocianate (AITC) experiment, HEK293 cells were plated in 24-well dishes at $2 \times 10^{5}$ cells/well and transiently transfected with Human TRPA1 in a pCMV6-AC-GFP vector (Viktorie Vlachova, Czech Academy of Sciences) using Lipofectamine 3000 (Thermo Fisher Scientific). For the transfection, $2 \mathrm{ml}$ of Lipofectamine 3000 was mixed with the DNA in DMEM plus Glutamax with 1\% FBS, a reduced serum media. Control cells received the media without the vector. Calcium imaging recordings took place $24 \mathrm{~h}$ after transfection. For the testosterone/WS-12 experiment, TRPM8 permanently transfected cells were obtained from Prof. Belmonte Laboratory (Instituto de Neurociencias, San Juan, Alicante, Spain). IMR90 fibroblast-like cells (CCL-186 ATCC, Virgina, USA) were seeded in $12 \mathrm{~mm}$ coverslips at 50.000 cells/well and were maintained in Minimum Essential Medium (MEM) enriched with 
$10 \% \mathrm{FBS}$ and penicillin/streptomycin $1 \%$ at $37^{\circ} \mathrm{C}$ in a $5 \% \mathrm{CO}_{2}$ atmosphere. IMR90 experiments were performed when confluence reached $50-60 \%$. were seeded in $12 \mathrm{~mm}$ coverslips at 50.000 cells/well and were maintained in Minimum Essential Medium (MEM) enriched with 10\% FBS and penicillin/streptomycin $1 \%$ at $37^{\circ} \mathrm{C}$ in a $5 \% \mathrm{CO}_{2}$ atmosphere. IMR90 experiments were performed when confluence reached $50-60 \%$. Cells were kept overnight in a hormone free medium, by eliminating FBS from the composition of the culture medium, substituting MEM or DMEM by no phenol red opti-MEM (Gibco 11058021).

2.9. Fluorescence $\mathrm{Ca}^{2+}$ imaging. Non-ratiometric calcium imaging experiments were conducted with the fluorescent indicator fluo4-AM (F14201, Thermo Fisher Scientific). Trigeminal neurons or Human Embrionic Kidney 293 (HEK293) cells (CRL-1573 ATCC) were incubated with 5 mM (6 mg/ml) fluo4-AM and $0.2 \% \mathrm{w} / \mathrm{v}$ pluronic acid (F-127, Thermo Fisher Scientific) for $60 \mathrm{~min}$ at $37^{\circ} \mathrm{C}$ in standard extracellular solution ( $\mathrm{NaCl} 140 \mathrm{mM}, \mathrm{KCl} 3 \mathrm{mM}, \mathrm{CaCl}_{2} 2.4 \mathrm{mM}, \mathrm{MgCl}_{2} 1.3 \mathrm{mM}, \mathrm{HEPES} 10 \mathrm{mM}$, and glucose $5 \mathrm{mM}$, adjusted to $\mathrm{pH} 7.4$ with $\mathrm{NaOH} 1 \mathrm{M}$ ). Afterwards, cells were washed with standard extracellular solution for at least 20 minutes. Fluorescence measurements were obtained on an inverted microscope (Axiovert 200/B, ZEISS) coupled to a Hamamatsu FLASH 4.0 LT camera (C11440-42U30, Hamamatsu, Sunayamacho, Japan). Before starting the experiment, an image of the microscopic field was obtained with transmitted light to identify cells with neuronal morphology. Then, Fluo4 was excited at $480 \mathrm{~nm}$ (excitation time 200 ms) with a rapid gating shutter (lambda-shutter 10/2 Sutter instruments, Novato, USA). Mean fluorescence intensity was recorded for each cell with HCimage DIA software (Hamamatsu Photonics) every 3 seconds. Calcium imaging recordings were performed at $35^{\circ} \mathrm{C}$. Response sizes after agonists were calculated by measuring peak minus basal values and divided by the positive control of the experiment, i.e. $\mathrm{KCl} 40 \mathrm{mM}$ in trigeminal primary cultures, and ionomicin $10 \mathrm{uM}$ in HEK293 and IMR90 human cell cultures. Responses were scored as positive if the increase in fluorescence was $>0.2$ arbitrary units. Substances dissolved in extracellular solution were delivered through a high-flow rate perfusion system controlled with an automatic system of valve clamps (PC-16 Bioscience Tools, S. Diego, USA). AITC (W203408, Merck) dissolved in 0.001\% DMSO, WS12 (3040/50, Tocris) dissolved in $0.001 \%$ ethanol, Nitroglicerin, Testosterone dissolved in $0.0001 \%$ ethanol, lonomicin (19657 Merck) and $\mathrm{KCl}$ were applied for periods of 10 to $30 \mathrm{~s}$, depending on the experiment. Cells were washed with extracellular solution between calcium responses for a period of at least $300 \mathrm{~s}$ to ensure recovery of basal fluorescence levels.

2.10. RNA extraction and RT-PCR. Trigeminal ganglia were isolated and frozen on dry ice until RNA extraction. Tissue was homogenized on ice using a polytron (Polytron PT 2000 Kinematica AG, Malters, Switzerland) and RNA was extracted with a TRIZOL (15596-026 Thermo Fisher Scientific) extraction method as previously described ${ }^{31}$. Briefly, chloroform was added to yield 2 phases, including one hydrophilic phase at the top containing RNA. Then after centrifugation the aqueous phase was mixed with isopropanol to precipitate RNA. The resulting pellet was washed with ethanol and air-dried. RNA purity and quantity were assessed by spectrophotometry (NanoDrop 2000, Thermo Fisher Scientific). Retrotranscription to cDNA was conducted with First Strand Synthesis Kit (K1612, Thermo Fisher 
Scientific) using dT primers. Primers for cDNA amplification were: TRPA1 fw 5'GCAGGTGGAACTTCATACCAA and rv 5'-CACTTTGCGTAAGTACCAGACTGG, TRPM8 fw 5'CTTTCTAAGCAATGGTATGGAG and rv 5'-GGTTTCTTCCTAAATGATACGAG, GAPDH fw 5'CCAATGTGTCCGTCGTGGATCT and rv 5'-GTTGAAGTCGCAG GAGACAACC. Relative expression values were obtained by applying the equation $2^{-(\triangle C T \text { sample- } \triangle C T G A P D H)}$.

2.11. Chemically-induced nocifensive behaviour (Formalin test). Mice were individually placed into transparent chambers and were habituated for $1 \mathrm{~h}$ before testing. Afterwards, $20 \mu \mathrm{l}$ of a $45 \% 2-$ Hydroxypropyl- $\beta$-cyclodextrin solution containing $5 \%$ formalin (F8775, Merck) and 0.6\% DMSO with or without $6 \mathrm{nmol}$ of WS-12 were injected subcutaneously into the plantar aspect of the right hind paw by using a Hamilton syringe (Hamilton Syringe Gastight ${ }^{T M}$ serie 1700 , TLL end, Merck) coupled to a 30-gauge needle. Nocifensive behavior (licking or biting of injected paw) was quantified in 5 min intervals during 60 min as previously described ${ }^{32}$. For the AMTB experiment, formalin was dissolved in saline.

2.12. Orchidectomy. Mice were anesthetized with a mixture of i.p. ketamine $(75 \mathrm{mg} / \mathrm{kg}$; Imalgene, 100 $\mathrm{mg} / \mathrm{ml}$, Boehringer Ingelheim, Ingelheim/Rhein, Germany) and xylazine (15 mg/kg, Merck) and a midline scrotal incision was made. The testes were exposed, and the vas deferens and testicular blood vessels were ligated with 2 tight knots of 6-0 black silk (8065195601, Alcon Cusi S.A., Barcelona, Spain). An incision was made between the 2 knots to remove testes and epididymis and the incision was closed with three additional square knots after ensuring haemostasis. Sham surgeries were performed similarly but the testicles were exposed and not ligated or removed. Subsequent nociceptive evaluations were conducted 3 weeks after surgeries.

2.13. Testosterone replacement treatment. Testosterone or vehicle (45\% 2 -Hydroxypropyl)- $\beta$-cyclodextrin in water) were placed into Alzet osmotic minipumps (Model 2004, $0.23 \mu \mathrm{l} / \mathrm{h}$ for 28 days) following manufacturer instructions. Minipumps were implanted subcutaneously between the scapulae under ketamine $(75 \mathrm{mg} / \mathrm{kg})$ - xylazine $(15 \mathrm{mg} / \mathrm{kg})$ anaesthesia. The pump was set to deliver vehicle or testosterone at an estimated dose of $6 \mu \mathrm{g} / \mathrm{h}$, based on previous works obtaining significant effects in orchidectomized mice ${ }^{23,33}$. Testing and NTG injections began after 3 days of minipump implantation.

2.14. Computational studies. Homology models of murine and human TRPM 8 channel were designed considering the structure of the TRPM8 from Ficedula albicollis, determined by cryo-electron microscopy at $4.1 \AA$ (Protein Data Bank code 6BPQ) (https://www.rcsb.org/). The sequence of murine TRPM8 (Uniprot Q8R455) or human TRPM8 (Uniprot Q7Z2W7) was completely modelled against the reference structure, following the standard protocol implemented by Yasara (version 20.12.24, http://www.yasara.org). Sequence alignments between murine or human TRPM8 and F. albicollis were performed with ClustalO from the European Bioinformatic Institute (EBI, https://www.ebi.ac.uk).

Blind docking experiments were carried out with AutoDock implemented in Yasara. WS12 (PubChem CID: 11266244), testosterone (PubChem CID: 6013), progesterone (PubChem CID: 5994) and estradiol (PubChem CID: 5757) structures were obtained from the National Center for Biotechnology Information 
(NCBI) PubChem database (https://pubchem.ncbi.nlm.nih.gov/). 800 docking runs with flexible ligands were fixed and results clustered around binding hot spots. By using the Assisted Model Building with Energy Refinement (AMBER03) force field, a simulated annealing optimization of the complexes was performed, which moved the structure to a stable energy minimum. The best binding energy in each cluster was saved and solutions grouped according to putative TRPM8 binding sites.

Local docking experiments focused in the menthol binding pocket were also performed with the murine and human TRPM8 model. A total of 50 runs were set with the side chain of critical residues in the menthol binding pocket kept flexible. Figures were drawn with open source PyMol (The PyMol Molecular Graphics System, version 1.8.2.0 Schrödinger, LLC, https://pymol.org/).

2.15. Statistical Analyses. Time courses of nociceptive behavioural data conducted in male and female mice were analysed using 2-way repeated measures ANOVA with time as within-subjects factor and NTG treatment or genotype as between-subject factors. The time courses involving orchidectomized animals were analysed with 3-way repeated measures ANOVA, with time as within-subjects factor and either NTG and orchidectomy or genotype and testosterone as between-subject factors. Levene's test of equality of error variances and Mauchly's sphericity tests were used to assess normality of the data and Bonferroni post-hoc pairwise comparisons were subsequently conducted when appropriate. Three-way ANOVA was also used to analyse the data of WS12 experiments (time point, WS12, NTG) whereas a within-design was chosen to analyse the effects of the AMTB doses in wild-type males recovered from sensitization (Friedman's test followed by Benjamini adjustment). A 3-way ANOVA was also used to analyse AMTB effects on NTG-exposed orchiectomized animals (Time point, Testosterone, Genotype). The time-course data of the chemically-induced nocifensive behaviour was analysed with repeated unadjusted t-tests to avoid assumptions of similar variances for the first and the second phases of the formalin test and posterior measurements. For the cellular studies, data normality was first assessed with the D'AgostinoPearson test. Comparisons of 2 groups were analysed accordingly with T-tests or Mann-Whitney-U tests. Comparisons of more than 2 groups were analysed with either One-way ANOVA followed by Bonferroni or Kruskal-Wallis followed by Mann-Whitney-U tests. RT-PCR and cellular data containing 2 factors were analysed with 2-way ANOVA followed by Bonferroni. Raw data can be found in the Supplementary Source Data File and results of the statistical tests are included in the Supplementary Statistical Results File.

\section{Results}

\subsection{Repeated NTG treatment induces a persistent hypersensitivity exclusively expressed in female mice.}

We implemented in our laboratory a modification of a previous model of chronic migraine induced by repeated NTG treatment ${ }^{4}$. Intraperitoneal administration (i.p.) of $10 \mathrm{mg} / \mathrm{kg} \mathrm{NTG}$ in a formulation of $10 \%$ ethanol, $20 \%$ propylene glycol in water revealed that the vehicle induced transient mechanical hypersensitivity in male and female mice (Suppl. Figure 1) and, therefore, it was discarded to prevent misinterpretation. Alternatively, a vehicle composed of $5 \%$ dextrose and $0.105 \%$ propylene glycol in water did not alter the animal nociception and was selected. We injected $10 \mathrm{mg} / \mathrm{kg} \mathrm{NTG}$ or vehicle i.p. every 
other day during 8 days to male and female mice, and mechanical sensitivity was assessed before and after each treatment. Measurements were extended up to 12 additional days after the last NTG injection (Figure 1A). Two hours after each NTG treatment, acute hypersensitivity was observed in male and female mice, whereas the vehicle did not induce nociceptive sensitization (Figures 1B and 1C, left panels, $\mathrm{P}<0.001$ treatment effect). Mechanical sensitivity assessed before NTG injections and after the end of the repeated treatment (Up to 20 days after beginning of the treatments, left panels of Figures 1B and 1C) showed a long-lasting hypersensitivity in male mice (Days 2-16, $P<0.05$ vs baseline and vehicle, Figure 1B) that returned to baseline values 8 days after the last NTG injection (Day 18, Figure 1B, nonsignificant vs. baseline or vs. vehicle). In marked contrast, female mice presented a persistent cutaneous hypernociception that was significant until the end of the experimental procedure $(P<0.05$ vs. baseline, vs. vehicle, Figure 1C). Thus, these data indicate a sexual dimorphism as males can fully recover from NTG sensitization, while females improve partially.

\subsection{Repeated NTG treatment induces long-lasting TRPA1-dependent hypersensitivity in female and male mice.}

Migraine-related pain produced by acute NTG treatment has been associated to TRPA1 activity in trigeminal ganglia of male mice ${ }^{3}$. We investigated the possible participation of TRPA1 on the model of chronic migraine. Wild-type and TRPA1 knockout mice received NTG injections and mechanical sensitivity was assessed (Figure 2A-B). As expected, wild-type mice showed acute hypersensitivity 2 hours after each NTG injection. On the contrary, this sensitization was absent in TRPA1 knockout mice of both sexes (Figure 2A-B, $P<0.001$ genotype effect, left panels), in agreement with a previous study describing lack of acute NTG sensitization in TRPA1 knockout males ${ }^{3}$. TRPA1 deletion also prevented the development of chronic hypersensitivity in males and females (Figure 2A-B, right panels).

To understand the role of TRPA1 in mediating NTG-induced sensitization in mice, we assessed TRPA1 mRNA expression and calcium imaging activity in trigeminal ganglia of male and female mice chronically exposed to NTG (Figures 2C and D). Samples from NTG-treated wild-type animals showed an increased TRPA1 mRNA expression regardless of the sex (Figure $2 \mathrm{C}, \mathrm{P}<0.001$ vs. vehicle). In parallel, trigeminal neuronal cultures of male and female mice revealed calcium transients in response to the TRPA1 agonist allyl isothiocyanate (AITC) at $70 \mu \mathrm{M}$ (Figure 2D). These responses were of similar magnitude in samples of both sexes and had higher intensity and duration in neurons of mice chronically exposed to NTG ( $\mathrm{P}<0.01$ vs. vehicle, Figure $2 \mathrm{E})$. The neuronal population responding to AITC was composed of small to medium size neurons (100 to $700 \mu \mathrm{m}^{2}$, Suppl. Fig. 2A). This increased TRPA1 responsiveness was also characterized by a higher percentage of cells showing significant activity (percentage of KCl-sensitive cells, $\mathrm{P}<0.001$ vs. vehicle, Suppl. Fig.2B), and the size of these responses was proportional to the percentage of sensitive cells (Suppl. Fig. 2C). Thus, the trigeminal cultures revealed small to medium size neurons with increased TRPA1 activity after chronic NTG treatment, regardless of the sex. 
To further investigate TRPA1 involvement on NTG sensitization, trigeminal neurons of naïve wild-type and TRPA1 knockout mice were cultured to assess calcium responses. These neurons were challenged with $100 \mu \mathrm{M}$ NTG followed by $70 \mu \mathrm{M}$ AITC (Figure $2 \mathrm{~F}$ ). A $15.7 \pm 3.2 \%$ of the cells responded to both stimuli in wild-type mice, while this percentage decreased drastically in TRPA1 knockouts $(0.6 \pm 0.3 \%, P<0.001$, Figure $2 \mathrm{G}$ ), revealing that NTG sensitized through TRPA1 activation in mice. To assess the translatability of this NTG activity to human TRPA1, NTG was applied to human TRPA1-transfected HEK293 cells and IMR90 fibroblast cells natively expressing this receptor (Figure 2H). Both cell types showed a dosedependent relationship for NTG-evoked calcium influx, whereas control HEK293 cells did not respond. Thus, NTG activated both human and murine TRPA1 channels.

TRPA1-mediated trigeminal sensitization may involve the release of the vasodilator peptide aCGRP ${ }^{6,8}$. This neuropeptide is an essential neurotransmitter for migraine neuroinflammation and pain ${ }^{34}$. Hence, we next examined NTG-induced aCGRP release in cultured trigeminal neurons through aCGRP immunofluorescence. A treatment with DD04107, an exocytosis inhibitor that interacts with the exocytosis-related protein SNAP- $25^{35}$ was used to investigate vesicular release. Control neurons exposed to NTG vehicle showed stronger aCGRP immunoreactivity than NTG-treated neurons (Figure 2I-J, $\mathrm{P}<0.001)$ suggesting vesicular aCGRP release after NTG. In contrast, neurons pre-treated with DD04107 showed significantly higher aCGRP immunoreactivity (Figure $2 \mathrm{~J}, \mathrm{P}<0.01 \mathrm{vs.}$ NTG+vehicle), indicating inhibition of the neuropeptide release after NTG. Collectively, these data indicate that TRPA1 activity was essential for acute and chronic NTG hypersensitivity, although it could not explain the observed sexual dimorphism.

\subsection{TRPM8 activity determines the recovery of normal sensitivity in male mice exposed to the model of chronic migraine.}

TRPM8 is a thermoTRP channel that has been signalled in the pathophysiology of chronic migraine. Thus, we next investigated the involvement of TRPM8 in NTG chronic sensitization. For this purpose, male and female wild-type and TRPM8 knockout mice were exposed to the chronic NTG treatment (Figure $3 A-B$ ). Wild-type males presented the expected sensitization (Figure $3 A, P<0.01$ vs. Baseline on days 8 and 15 ) that was resolved by the end of the experimental procedure (day 20, Figure $3 A$ ). Noteworthy, TRPM8 knockout males maintained a persistent sensitization that was significant until the last day of measurements, akin to the persistent sensitization observed in females $(P<0.01$ vs. Baseline and wildtype, Right panel of Figures $3 \mathrm{~A}$ and $3 \mathrm{~B}$ ). This finding revealed a protective function of TRPM8 in males subjected to NTG-mediated chronic sensitization and signalled a potential role of this channel in sex dimorphism in the model of chronic migraine.

To investigate the involvement of TRPM8 in sex dimorphism, we next analysed its expression in trigeminal ganglia of male and female wild-type mice chronically exposed to NTG. TRPM8 mRNA values were similar regardless of treatment and sex (Figure $3 \mathrm{C}$ ). Functionally, perfusion with the selective and potent TRPM8 agonist WS12 (500 nM) elicited calcium transients of similar morphology and size in both sexes, after chronic vehicle or NTG (Figures 3D, 3E). WS12 activated $8 \pm 2.1 \%$ of the cultured 
neurons (Supplementary Figure 3A), which were small size cells $\left(50-400 \mu \mathrm{m}^{2}\right.$, Supplementary Figures 3BC). This percentage was similar in samples of vehicle and NTG-treated mice. Hence, trigeminal cultures showed that TRPM8 activity and expression were similar between sexes and after the NTG treatment.

Given this similar functionality, we conducted additional experiments to test the antinociceptive efficacy of WS12 in wild-type female mice sensitized after chronic NTG. On day 20, females received WS12 or its vehicle. The observed responses were highly variable and nonsignificant results were found after 10 $\mathrm{mg} / \mathrm{kg}$ WS12 (i.p.), a dose with reported antinociceptive efficacy in male mice ${ }^{36}$ (Figure 3F). In a separate experiment, we tested the effect of lower $(5 \mathrm{mg} / \mathrm{kg}$, Supplementary Figure $4 \mathrm{~A})$ and higher doses of WS12 (20 mg/kg, Supplementary Figure 4B) in wild-type and TRPM8 knockout females. An antinociceptive trend was observed with the highest dose (Supplementary Figure 4B, $P=0.053$ vs. vehicle), whereas the lowest dose was completely ineffective. WS-12 also showed efficacy when administered in a vehicle containing $5 \%$ ethanol and $45 \%$ cyclodextrin, however possible antinociceptive effects of this vehicle were detected (Supplementary Figure 4C). To further characterize the in vivo activity of WS12 in females, we decided to examine the pain-relieving effect of TRPM8 activity on the formalin pain model, another TRPA1-dependent pain model ${ }^{37}$. As illustrated in Figure 3G, WS12 induced significant antinociception in the acute phase, whereas the late phase was unaltered, revealing a short-lasting effect of this compound.

To further investigate the function of TRPM8 in wild-type males, mice previously exposed to NTG that had recovered their baseline sensitivity were exposed to increasing doses of the TRPM8 blocker AMTB (Figure 3G). This compound precipitated a significant re-sensitization when administered at 10 or 15 $\mathrm{mg} / \mathrm{kg}(\mathrm{P}<0.05 \mathrm{vs}$. vehicle, Figure $3 \mathrm{H})$ in this model. Interestingly, similar results were obtained in male mice previously subjected to the formalin test. Thus, male mice treated with AMTB after the extinction of formalin-induced nocifensive activity showed a significant reinstatement of licking behaviour evident 30 min after administration of the compound ( $P<0.05$ vs. vehicle, Figure $3 \mathrm{I})$. Altogether, these results suggest the presence of endogenous TRPM8 activity with pain-relieving function in male mice.

\subsection{Testosterone activates TRPM8 to resolve mechanical sensitization in male mice exposed to the model of chronic migraine.}

Testosterone has been suggested as an endogenous TRPM8 agonist ${ }^{22}$ and shows antinociceptive functions in mice $^{23}$. We hypothesized that this endogenous androgen could have a protective function in the mouse model of chronic migraine. To address this question, mice were first subjected to a sham surgery or to an orchidectomy to deplete gonadal testosterone. Once their nociceptive sensitivity was restored the animals received the NTG treatment (Figure 4A). Acute NTG produced similar hypernociception in sham and orchidectomized animals (Figure 4A, left panel, $\mathrm{P}<0.001$ vs. vehicle). In contrast, the repeated NTG evidenced a persistent chronic sensitization selectively expressed in orchidectomized animals (Figure 4A, right panel, $\mathrm{P}<0.01 \mathrm{vs}$. sham), indicating possible protective role of testosterone. 
To investigate whether testosterone could have TRPM8-mediated antinociceptive effect in males, wildtype and TRPM8 knockout males were orchidectomized and received subcutaneous osmotic pumps filled with testosterone or its vehicle (cyclodextrin $45 \%$ in water). Afterwards, all mice were chronically treated with NTG, and their mechanical sensitivity was assessed. Testosterone induced complete recovery of mechanical thresholds in wild-type mice (Figure 4B, right panel, day 20, $P<0.001$ vs. Vehicle wild-type, nonsignificant vs. baseline), while vehicle-treated animals remained sensitized by the end of the experiment (Figure 4B, right pane, $P<0.001$ vs. baseline on day 20). Remarkably, TRPM8 knockout mice lacked this restorative effect of testosterone (Figure 4B, right panel, day 20, $\mathrm{P}<0.001$ vs. wild-type, $P<0.001$ vs. baseline), although an antinociceptive effect independent of TRPM8 activity was also evidenced in knockouts (Figure 4B, right panel, day 20, P<0.05 vs. vehicle TRPM8 knockout). To further clarify the involvement TRPM8 in the protective effect of testosterone, AMTB was administered to all mice after the nociceptive measurement on day 20 (Figure 4C). A significant drop in the mechanical thresholds was selectively observed in wild-type animals treated with testosterone (Figure $4 C, P<0.01$ vs. values before AMTB), whereas mice receiving vehicle and knockouts showed unaltered mechanosensitivity. These results revealed a prominent testosterone-TRPM8 antinociception in males.

Next, to determine whether testosterone could have rapid antinociceptive effects we treated NTG-exposed wild-type and TRPM8 knockout females with a single subcutaneous administration of $1 \mathrm{mg} / \mathrm{kg}$ testosterone or its vehicle (2.5\% DMSO in corn oil, Figure 4D). A significant testosterone-induced antinociception was observed selectively in wild-type mice ( $P<0.05$ vs. pre-treatment values, $P<0.05$ vs. testosterone-treated TRPM8 knockouts), whereas TRPM8 knockout females showed a trend for a hypersensitivity. Overall, the present behavioural results suggest nongenomic antinociceptive effects of testosterone through TRPM8.

We next investigated if testosterone activated TRPM8 channels in trigeminal cultures as a mechanism to account for the protective role of the androgen. Trigeminal cultures of wild type mice showed calcium transients in response to $10 \mathrm{pM}$ testosterone (Figure 4E). Cells responding to testosterone also presented calcium transients in response to $500 \mathrm{nM}$ WS12 (7.9 $0.8 \%$ of KCl-sensitive cells; Figure 4E). These responses were abolished in neural cultures from TRPM8 knockout animals tested side-by-side (Figures $4 \mathrm{E}, \mathrm{P}<0.001$ vs. wild-type), revealing a testosterone activity through TRPM8 in trigeminal neurons. Previous studies described structural and functional differences between murine and human TRPM ${ }^{38}$. To evaluate this possibility, we assessed testosterone activity on HEK293 cells constitutively expressing murine or human TRPM8 (Figure 4F). Similar calcium transients were elicited after testosterone $10 \mathrm{pM}$ in cells expressing human or murine TRPM8, whereas control HEK293 cells lacked this response (Figure $4 \mathrm{~F})$. Altogether, the present data reveal a testosterone activity on murine and human TRPM8 that exerts antinociceptive restorative effects on the mouse model of chronic migraine.

\section{Discussion}

The most salient contribution of our study is the discovery of a novel role of TRPM8 as an androgen receptor that provides antinociceptive resilience and favours recovery in a mouse model of NTG-induced 
chronic migraine that produces similar acute mechanosensitivity in males and females but persistent hypersensitivity exclusively in females. Testosterone by activating TRPM8 channels drives a sexual dimorphism characterized by recovery of normal sensitivity in males. The lack of this protective mechanism in females leads to a persistent mechanical hypersensitivity. Noteworthy, our model of chronic migraine mimics the sexual dimorphism observed in humans, characterized by stronger transitions to chronic sensitization in women ${ }^{24,39}$ and possible higher resilience in men. Previous studies also described higher female sensitivity in models of formalin-inflammatory pain ${ }^{40}$ and models of persistent pain such as stress-induced visceral hypersensitivity or muscle pain after repeated saline injections and forced activity ${ }^{23,40}$ Overall, these findings suggest that the exposure to repeated noxious insults such as ongoing inflammation and stress-related stimuli favour the perpetuation of painful responses in females, whereas males show higher resilience and are able of reinstating their normal sensitivity.

In our model of chronic migraine, NTG provokes mechanical hypersensitivity by signalling through the TRPA1 channel, although this thermoTRP is not involved in the sex dimorphism observed. Indeed, repeated NTG exposure induced similar TRPA1 mRNA overexpression in trigeminal ganglia of male and female mice. Increased expression translated into exacerbated TRPA1 activity and trigeminal cultures of males and females chronically exposed to NTG presented stronger and longer-lasting TRPA1 activity in response to the specific agonist AITC. In line with our results, previous research described TRPA1-specific neuronal responses after acute $\mathrm{NTG}^{3}$, and male rodents exposed to NTG were sensitive to TRPA1 antagonism ${ }^{41}$. Our findings reveal an essential participation of TRPA 1 for the development of chronic NTG hypersensitivity in male and female mice, in agreement with studies showing TRPA1 involvement in persistent pain sensitizations $\mathbf{8}^{8,42-44}$.

The NTG-TRPA1 mechanism involved in promoting the mechanical hypersensitivity relies on the release for aCGRP from trigeminal neurons ${ }^{6,8}$, in agreement with the critical role of this neuropeptide in the aetiology of chronic migraine $e^{9}$. Our data further substantiate this tenet as sensitivity to the exocytosis inhibitor DD04107 revealed a mechanism involving large dense core vesicles and vesicular fusion protein SNAP-25 $5^{35}$. Notably, the data obtained with DD04107 suggest a potential efficacy of this peptide for the treatment of chronic migraine through inhibition of aCGRP release, similar to botulinum neurotoxin ${ }^{10}$. Accordingly, we propose antagonistic roles of TRPA1 and TRPM8 activities determined by the continuous exposure to exogenous and endogenous agonists. Namely, nitric oxide derived from the treatment with NTG elicits a hyperalgesic state via TRPA1 stimulation both in males and females, whereas testosterone exerts an antinociceptive role through high-affinity interactions with TRPM8.

Notably, our results in TRPM8 knockout mice subjected to chronic NTG sensitization reveal a protective function of TRPM8. In line with our data, TRPM8 stimulation showed efficacy alleviating thermal hyperalgesia and nocifensive behaviours in models of headache-related pain ${ }^{45,46}$. A protective function was also described in models of noxious heat or chemically-induced pain such as the injection of capsaicin or the TRPA1 agonist acrolein ${ }^{18,28}$. TRPM8 stimulation also provided alleviation of mechanical 
and cold sensitivity in models of chronic neuropathic pain ${ }^{47,48}$. In accordance with these preclinical findings, TRPM8 agonists have been used medicinally for alleviating a variety of pain conditions including migraine ${ }^{13,14,49,50}$, although using these compounds is not a first-line treatment for this clinical condition ${ }^{10}$. Contradictory results were also published showing pro-algesic effects of TRPM8 agonists on migraine, although the use of TRPM8 agonists with partial effect on TRPA1 such as icilin could have yielded misleading results ${ }^{51}$. Similarly, we only observed an antinociceptive trend after administration of high WS12 doses in female mice treated with NTG. The absence of a prominent antinociception after WS12 could be associated to the potent and short-lasting effect of this compound, as suggested by the results obtained in the formalin test where significant effects lasted only $5 \mathrm{~min}$ in the acute phase. In agreement, calcium-dependent desensitization of TRPM8 is described after application of the canonical TRPM8 agonists menthol, icilin or WS12 ${ }^{15,52}$. In our von Frey experiments, reliable assessment of mechanosensitivity requires spaced and sequential application of von Frey filaments during periods of 15-30 min. Hence, mechanosensitivity could not be precisely assessed at that specific temporal resolution and a transient effect of WS12 could have been overlooked. Short-lasting pain-relieving responses to menthol and its derivatives can be found clinically and were described elsewhere ${ }^{49}$.

The protective role of TRPM8 was ratified by the ATMB-induced resensitization of males already recovered from the chronic NTG treatment. This protective TRPM8 activity was corroborated in males previously subjected to the classical formalin test and is compatible with a latent pain sensitization masked by tonic TRPM8 activity. The development of latent pain sensitizations or hyperalgesic priming has been related to the establishment of chronic pain conditions ${ }^{53-55}$ and an endogenous opioid tone has been associated with this tonic pain inhibition ${ }^{54}$. In line with studies on acute pain models demonstrating involvement of opioidergic activity in TRPM8-induced antinociception ${ }^{36,56}$, our results suggest the interest of investigating opioid-TRPM8 interactions for the promotion of pain resilience.

Noteworthy, testosterone application provided rapid TRPM8-mediated antinociception in females. In line with this finding, orchidectomized males showed persistent sensitization after chronic NTG similar to females. Previous studies also described decreased ability of orchidectomized males in restoring normal mechanosensitivity after inflammatory or stress-related insults $23,40,57$. These studies elucidated testosterone antinociceptive mechanisms including transformation to di-hydrotestosterone and binding to androgen receptor ${ }^{40}$, down-regulation of anti-opioid neurotransmitter Brain-derived Neurotrophic Factor $(B D N F)^{57}$ or modulation of serotonin transporters ${ }^{23}$. In our study, orchidectomized wild-type males exposed to testosterone recovered their normal sensitivity after cessation of the NTG treatment, and this effect was largely dependent on TRPM8. These data reveal a novel function of TRPM8 providing endogenous pain relief in males though testosterone stimulation. Male-specific alterations have been previously observed in TRPM8 knockouts, including delayed cold acclimation and lower bone mineral density, similar to females of either genotype that showed this same phenotype ${ }^{58}$. Interestingly, these features are also tightly linked to the activity of sexual steroids ${ }^{59}$. We observed rapid antinociceptive effects of testosterone in NTG-exposed females, in a time frame in which acute testosterone anxiolytic 
effects are also found ${ }^{60}$. In agreement, preclinical works show pain-relieving testosterone efficacy in male and female rodent models of acute and chronic pain ${ }^{23,40,57}$. Consistently, clinical treatments with testosterone provide pain alleviation ${ }^{61}$, and testosterone levels of men and women are inversely proportional to pain perception ${ }^{62,63}$ including migraine pain ${ }^{25}$. However, hormonal treatments are subjected to tight regulation and have transcriptional effects and deleterious consequences that limit their use ${ }^{64}$.

Our trigeminal cultures revealed TRPM8 calcium transients in response to physiological picomolar concentrations of testosterone ${ }^{65}$ that were absent in cultures of TRPM8 knockouts. In agreement, TRPM8 testosterone activity was also elucidated in somatosensory neurons through blockade with selective TRPM8 antagonism ${ }^{22}$. Thus, testosterone shows rapid non-genomic effects on primary afferent neurons through TRPM8. While WS12 in the nanomolar range elicits stronger calcium transients than picomolar concentrations of testosterone ${ }^{65}$, at the picomolar level TRPM8 agonists such as menthol, icilin or WS12 lack significant calcium activity ${ }^{22}$. These results and the behavioural data strongly correlate with computational docking studies revealing higher affinity for testosterone-TRPM8 interactions when compared to WS12-TRPM8 binding (Figure 5AB and Supplementary Figure 5AB). Interestingly, the results obtained both in murine and human TRPM8 models indicate that testosterone most likely binds to the active pocket described for WS12 and menthol ${ }^{52,66}$. Interestingly, female hormones such as estradiol or progesterone show lower TRPM8 affinity than testosterone (Figures 5CD and Supplementary Figure 5CD). In this line TRPM3, another TRP channel of the melastatin family, has been established as a thermoreceptor ${ }^{67}$ that also responds to sexual hormones such as pregnenolone and progesterone ${ }^{68}$, suggesting a dual role of these protein types as detectors of both exogenous and endogenous stimuli. Our data suggest a direct testosterone-TRPM8 interaction that could be helpful for the design of novel compounds mimicking this channel agonist activity without the unwanted effects of hormonal treatments. Additional less popular docking solutions include regions without described impact on TRPM8 functionality, and mutagenesis studies will be needed to clarify the specific role of these interactions.

In conclusion, a high sensitization level of females has been found in a model that reproduces the sexual dimorphism and the enhanced cutaneous sensitivity associated with chronic migraine in humans ${ }^{5}$. This type of chronic sensitization could be aggravated after recurrent exposure to environmental factors involving TRPA1 stimulation such as pungent substances, oxidative stress or cyclic proinflammatory events ${ }^{69}$. The present data reveals that the difference between males and females is mainly due to an increased nociceptive resilience of males. This effect relies partly on TRPM8 activity in response to endogenous testosterone, an androgenic hormone that is present at much higher levels in male individuals. Hence, novel molecules mimicking testosterone activity on TRPM8 could lack the unwanted effects of hormonal treatments and may provide effective pain relief for individuals with low testosterone levels of any gender. The sexual dimorphism described here is based on a restorative natural process selectively expressed in males and encourages further investigation on pathophysiological processes 
promoting resilience. In addition, our data endorse the interest of analgesic drugs inhibiting TRPA1 activity for the treatment or prevention of migraine and pain syndromes characterized by enhanced mechanosensitivity in males and females ${ }^{43,70,71}$, although careful modulation may be desirable since TRPA1 shows neuroprotective effects as a hypoxia sensor that drives vasodilation and reduces ischemic damage ${ }^{72}$.

\section{Declarations}

\section{Author Contributions}

D.A.A. conducted calcium imaging, cell culture, immunofluorescence, RT-PCR experiments, performed formalin behavioural assays, designed experiments, analysed the data, wrote and edited the first draft of the manuscript. D.C. conducted all behavioural assays, performed surgeries, analysed the data, conceptualized and designed experiments, coordinated in vivo, in vitro and computerized experiments and wrote and edited the first draft of the manuscript. J.D.L. executed and analysed docking experiments and wrote the first draft of the manuscript. G.F.B. supervised and executed docking experiments and revised and edited the manuscript. A.F.C. supervised and designed experiments, conceptualized the project, revised and edited the manuscript and provided funding. A.F.M. supervised and designed experiments, conceptualized the project, wrote, revised and edited the manuscript and provided funding.

\section{Conflicts of interest statement}

The authors have no conflict of interest to declare.

\section{Data Availability}

Raw behavioural and molecular data can be found in the Supplementary Source Data File and results of the statistical tests are included in the Supplementary Statistical Results File. Raw data of calcium imaging studies are available from the corresponding author upon reasonable request and individual data points are provided in figures whenever possible.

\section{Acknowledgements}

Financial support of Spanish Ministry of Science, Innovation and Universities RTI2018-097189-B-C21 to A.F.C. and A.F.M. and Universidad Miguel Hernández de Elche UMH-PAR2019 to A.F.C. and A.F.M. D.A.A. SAF2015-66275-C2-1-R. J.D.L. PRE2019-091317. Authors thank Gema Osuna Tenorio and José Manuel Serrano García for their help and technical expertise. We thank Dr. F. Viana for providing the TRPM8 knockout mice. 


\section{References}

1. Safiri S, Pourfathi H, Eagan A, Mansournia MA, Khodayari MT, Sullman MJM, Kaufman J, Collins G, Dai H, Bragazzi NL, K. A. Global, regional, and national burden of migraine in 204 countries and territories, 1990 to 2019. Pain (2021).

2. Demartini, C. et al. Nitroglycerin as a comparative experimental model of migraine pain: From animal to human and back. Prog. Neurobiol. 177, 15-32 (2019).

3. Marone, I. M. et al. TRPA1 / NOX in the soma of trigeminal ganglion neurons mediates migrainerelated pain of glyceryl trinitrate in mice. Brain 141, 2312-2328 (2018).

4. Pradhan, A. et al. Characterization of a novel model of chronic migraine. Pain 155, 269-274 (2014).

5. Louter, M. A. et al. Cutaneous allodynia as a predictor of migraine chronification. Brain 136, 34893496 (2013).

6. Nassini, R. et al. The 'headache tree' via umbellulone and TRPA1 activates the trigeminovascular system. Brain 135, 376-390 (2012).

7. Benemei, S. \& Dussor, G. TRP channels and migraine: Recent developments and new therapeutic opportunities. Pharmaceuticals 12, 1-17 (2019).

8. De Logu, F. et al. Schwann cells expressing nociceptive channel TRPA1 orchestrate ethanol-evoked neuropathic pain in mice. J. Clin. Invest. 129, 5424-5441 (2019).

9. Charles, A. \& Pozo-Rosich, P. Targeting calcitonin gene-related peptide: a new era in migraine therapy. The Lancet 394, 1765-1774 (2019).

10. Ashina, M. et al. Migraine: integrated approaches to clinical management and emerging treatments. The Lancet 397, 1505-1518 (2021).

11. Chasman, D. I. et al. Genome-wide association study reveals three susceptibility loci for common migraine in the general population. in Nature Genetics 43, 695-698 (Nat Genet, 2011).

12. Chasman, D. I. et al. Selectivity in Genetic Association with Sub-classified Migraine in Women. PLoS Genet. 10, (2014).

13. Borhani Haghighi, A. et al. Cutaneous application of menthol $10 \%$ solution as an abortive treatment of migraine without aura: A randomised, double-blind, placebo-controlled, crossed-over study. Int. J. Clin. Pract. 64, 451-456 (2010).

14. St. Cyr, A. et al. Efficacy and tolerability of STOPAIN for a migraine attack. Front. Neurol. 6, (2015).

15. McKemy, D. D., Neuhausser, W. M. \& Julius, D. Identification of a cold receptor reveals a general role for TRP channels in thermosensation. Nature 416, 52-58 (2002).

16. Peier, A. M. et al. A TRP channel that senses cold stimuli and menthol. Cell 108, 705-715 (2002).

17. Bautista, D. M. et al. The menthol receptor TRPM 8 is the principal detector of environmental cold. 448, 4-9 (2007).

18. Dhaka, A. et al. TRPM8 Is Required for Cold Sensation in Mice. Neuron 54, 371-378 (2007). 
19. Pérez De Vega, M. J., Gómez-Monterrey, I., Ferrer-Montiel, A. \& González-Muñiz, R. Transient Receptor Potential Melastatin 8 Channel (TRPM8) Modulation: Cool Entryway for Treating Pain and Cancer. Journal of Medicinal Chemistry 59, 10006-10029 (2016).

20. Liu, Y. et al. TRPM8 channels: A review of distribution and clinical role. European Journal of Pharmacology 882, 173312 (2020).

21. Ordás, P. et al. Expression of the cold thermoreceptor TRPM8 in rodent brain thermoregulatory circuits. J. Comp. Neurol. 529, 234-256 (2021).

22. Asuthkar, S. et al. The TRPM8 protein is a testosterone receptor: II. Functional evidence for an ionotropic effect of testosterone on TRPM8. J. Biol. Chem. 290, 2670-2688 (2015).

23. Lesnak, J. B., Inoue, S., Lima, L., Rasmussen, L. \& Sluka, K. A. Testosterone protects against the development of widespread muscle pain in mice. Pain 161, 2898-2908 (2020).

24. Rosen, S., Ham, B. \& Mogil, J. Sex differences in neuroimmunity and pain. J. Neurosci. Res. 95, 500508 (2017).

25. Shields, L. B. E., Seifert, T., Shelton, B. J. \& Plato, B. M. Testosterone levels in men with chronic migraine. Neurol. Int. 11, 8079 (2019).

26. Kwan, K. Y. et al. TRPA1 Contributes to Cold, Mechanical, and Chemical Nociception but Is Not Essential for Hair-Cell Transduction. Neuron 50, 277-289 (2006).

27. Zimmermann, M. Ethical considerations in relation to pain in animal experimentation. Acta Physiol. Scand. Suppl. 554, 221-33 (1986).

28. Wang, S. L., Dai, Y., Liu, X. L. \& Zhang, W. S. TRP channels and migraine. Chinese Pharmacological Bulletin 29, 1352-1354 (2013).

29. Karashima, Y. et al. Bimodal action of menthol on the transient receptor potential channel TRPA1. J. Neurosci. 27, 9874-9884 (2007).

30. Chaplan, S. R. et al. Quantitative assessment of tactile allodynia in the rat paw. J. Neurosci. Methods 53, 55-63 (1994).

31. Chomzynski, P. Single-Step Method of RNA Isolation by Acid Guanidinium Thiocyanate-PhenolChloroform Extraction. Anal. Biochem. 162, 156-159 (1987).

32. McNamara, C. R. et al. TRPA1 mediates formalin-induced pain. Proc. Natl. Acad. Sci. U. S. A. 104, 13525-13530 (2007).

33. Zhao, R. et al. A GPR174-CCL21 module imparts sexual dimorphism to humoral immunity. Nature 577, 416-420 (2020).

34. Denner, A. C., Vogler, B., Messlinger, K. \& De Col, R. Role of transient receptor potential ankyrin 1 receptors in rodent models of meningeal nociception - Experiments in vitro. Eur. J. Pain (United Kingdom) 21, 843-854 (2017).

35. Camprubí-Robles, M., Planells-Cases, R., Ferrer-Montiel, A. \& Robles, C. Differential contribution of SNARE-dependent exocytosis to inflammatory potentiation of TRPV1 in nociceptors. FASEB J. 23, 3722-3733 (2009). 
36. Liu, B. et al. TRPM8 is the principal mediator of menthol-induced analgesia of acute and inflammatory pain. Pain 154, 2169-2177 (2013).

37. McNamara, C. R. et al. TRPA1 mediates formalin-induced pain. Proc. Natl. Acad. Sci. U. S. A. 104, 13525-13530 (2007).

38. Journigan, V. B. et al. Structural and in Vitro Functional Characterization of a Menthyl TRPM8 Antagonist Indicates Species-Dependent Regulation. ACS Med. Chem. Lett. 12, 758-767 (2021).

39. Bartley, E. J. \& Fillingim, R. B. Sex differences in pain: A brief review of clinical and experimental findings. Br. J. Anaesth. 111, 52-58 (2013).

40. Fanton, L. E., Macedo, C. G., Torres-Chávez, K. E., Fischer, L. \& Tambeli, C. H. Activational action of testosterone on androgen receptors protects males preventing temporomandibular joint pain. Pharmacol. Biochem. Behav. 152, 30-35 (2017).

41. Demartini, C. et al. The role of the transient receptor potential ankyrin type-1 (TRPA1) channel in migraine pain: evaluation in an animal model. J. Headache Pain 18, 94 (2017).

42. Wei, H. et al. Spinal transient receptor potential ankyrin 1 channel contributes to central pain hypersensitivity in various pathophysiological conditions in the rat. Pain 152, 582-591 (2011).

43. Liu, C. et al. A Non-covalent Ligand Reveals Biased Agonism of the TRPA1 Ion Channel. Neuron 109, 273-284 (2021).

44. Martínez-Rojas, V. A. et al. Peripheral and spinal TRPA1 channels contribute to formalin-induced longlasting mechanical hypersensitivity. J. Pain Res. 11, 51-60 (2018).

45. Kayama, Y. et al. Functional interactions between transient receptor potential M8 and transient receptor potential V1 in the trigeminal system: Relevance to migraine pathophysiology. Cephalalgia 38, 833-845 (2018).

46. Ren, L., Dhaka, A. \& Cao, Y. Q. Function and postnatal changes of dural afferent fibers expressing TRPM8 channels. Mol. Pain 11, 37 (2015).

47. Knowlton, W. et al. A sensory-labeled line for cold: TRPM8-expressing sensory neurons define the cellular basis for cold, cold pain, and cooling-mediated analgesia. J. Neurosci. 33, 2837-2848 (2013).

48. Patel, R. et al. Anti-hyperalgesic effects of a novel TRPM8 agonist in neuropathic rats: A comparison with topical menthol. Pain 155, 2097-2107 (2014).

49. Eccles, R. Menthol and Related Cooling Compounds. Journal of Pharmacy and Pharmacology 46, 618-630 (1994).

50. Pergolizzi, J. V., Taylor, R., LeQuang, J. A. \& Raffa, R. B. The role and mechanism of action of menthol in topical analgesic products. Journal of Clinical Pharmacy and Therapeutics 43, 313-319 (2018).

51. Burgos-Vega, C. et al. Meningeal transient receptor potential channel M8 activation causes cutaneous facial and hindpaw allodynia in a preclinical rodent model of headache. Cephalalgia 36, 185-193 (2016). 
52. Yin, Y. et al. Structural basis of cooling agent and lipid sensing by the cold-activated TRPM8 channel. Science (80-). 363, eaav9334 (2019).

53. Reichling, D. B. \& Levine, J. D. Critical role of nociceptor plasticity in chronic pain. Trends in Neurosciences 32, 611-618 (2009).

54. Corder, G. et al. Constitutive $\mu$-opioid receptor activity leads to long-term endogenous analgesia and dependence. Science (80-). 341, 1394-1399 (2013).

55. Cabañero, D., Campillo, A., Célérier, E., Romero, A. \& Puig, M. M. Pronociceptive effects of remifentanil in a mouse model of postsurgical pain: effect of a second surgery. Contro/ 111, 1334-1345 (2009).

56. Shapovalov, G. et al. Opiates modulate thermosensation by internalizing cold receptor TRPM8. Cell Rep. 4, 504-515 (2013).

57. Ji, Y., Hu, B., Li, J. \& Traub, R. J. Opposing Roles of Estradiol and Testosterone on Stress-Induced Visceral Hypersensitivity in Rats. J. Pain 19, 764-776 (2018).

58. Carvalho, A. L. et al. TRPM8 modulates temperature regulation in a sex-dependent manner without affecting cold-induced bone loss. PLoS One 16, e0231060 (2021).

59. Khosla, S. \& Monroe, D. G. Regulation of bone metabolism by sex steroids. Cold Spring Harbor Perspectives in Medicine 8, a031211 (2018).

60. Frye, C. A., Koonce, C. J., Edinger, K. L., Osborne, D. M. \& Walf, A. A. Androgens with activity at estrogen receptor beta have anxiolytic and cognitive-enhancing effects in male rats and mice. Horm. Behav. 54, 726-734 (2008).

61. White, H. D. \& Robinson, T. D. A novel use for testosterone to treat central sensitization of chronic pain in fibromyalgia patients. International Immunopharmacology 27, 244-248 (2015).

62. Bartley, E. J. et al. Natural Variation in Testosterone is Associated with Hypoalgesia in Healthy Women. Clin. J. Pain 31, 730-739 (2015).

63. Choi, J. C., Chung, M. I. \& Lee, Y. D. Modulation of pain sensation by stress-related testosterone and cortisol. Anaesthesia 67, 1146-1151 (2012).

64. Bianchi, V. E. et al. The role of androgens in women's health and wellbeing. Pharmacological Research 171, 105758 (2021).

65. Yoo, H. S. \& Napoli, J. L. Quantification of Dehydroepiandrosterone, $17 \beta$-Estradiol, Testosterone, and Their Sulfates in Mouse Tissues by LC-MS/MS. Anal. Chem. 91, 14624-14630 (2019).

66. Yin, Y. et al. Structure of the cold- And menthol-sensing ion channel TRPM8. Science (80-). 359, 237241 (2018).

67. Paricio-Montesinos, R. et al. The Sensory Coding of Warm Perception. Neuron 106, 830-841.e3 (2020).

68. Majeed, Y. et al. Pregnenolone sulphate-independent inhibition of TRPM3 channels by progesterone. Cell Calcium 51, 1-11 (2012).

69. Giorgi, S., Nikolaeva-Koleva, M., Alarcón-Alarcón, D., Butrón, L. \& González-Rodríguez, S. Is TRPA1 burning down TRPV1 as druggable target for the treatment of chronic pain? International Journal of 
Molecular Sciences 20, 2906 (2019).

70. Ferrer-Montiel, A. et al. Advances in modulating thermosensory TRP channels. Expert Opinion on Therapeutic Patents 22, 999-1017 (2012).

71. Souza Monteiro de Araujo, D., Nassini, R., Geppetti, P. \& De Logu, F. TRPA1 as a therapeutic target for nociceptive pain. Expert Opinion on Therapeutic Targets 24, 997-1008 (2020).

72. Pires, P. W. \& Earley, S. Neuroprotective effects of trpa1 channels in the cerebral endothelium following ischemic stroke. Elife 7, e35316 (2018).

\section{Figures}

A
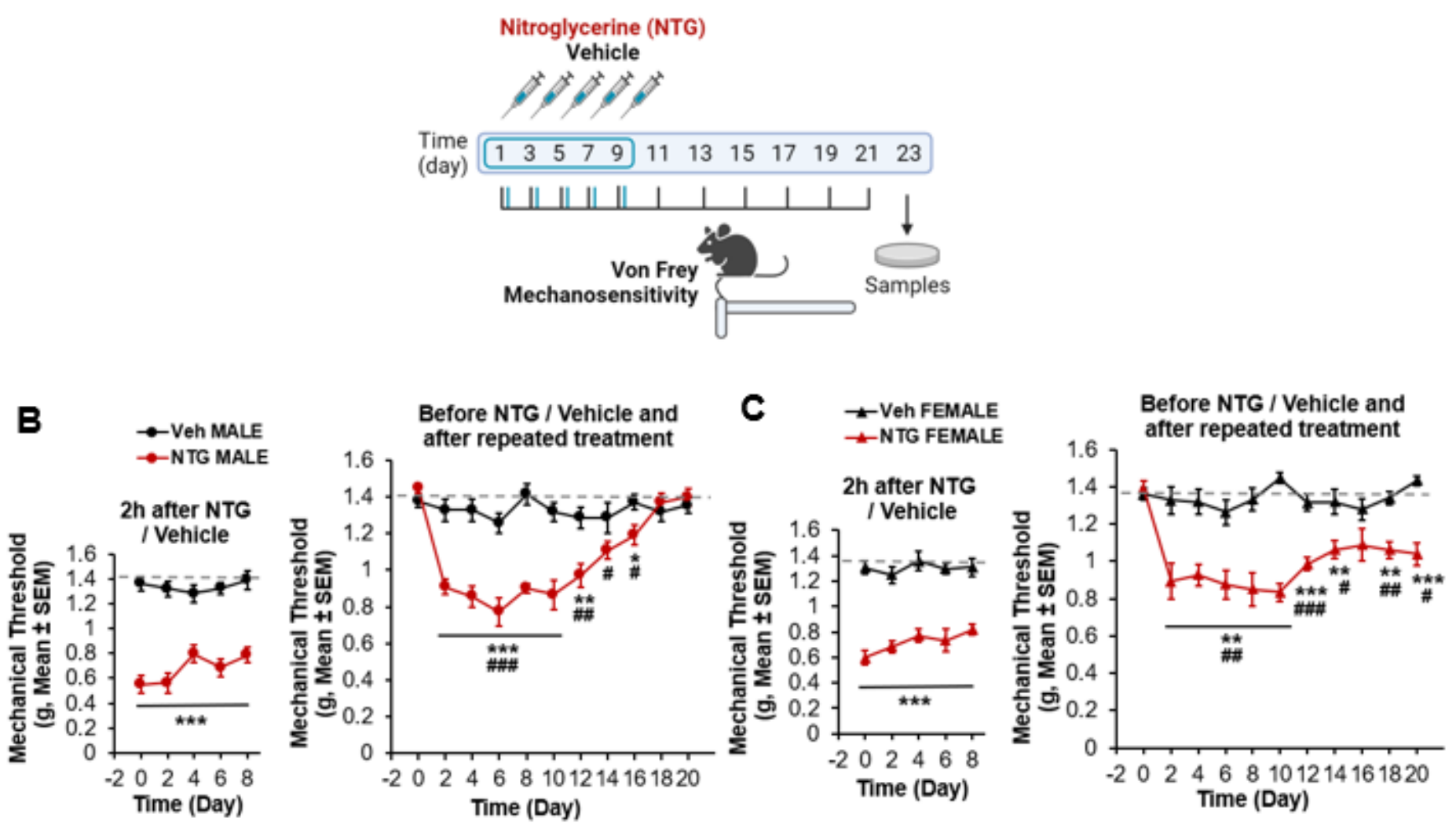

Figure 1

Chronic nitroglycerin administration induces generalized long-lasting mechanical hypersensitivity in male and female mice. A Model of chronic migraine induced by 5 intraperitoneal injections of nitroglycerin 10 $\mathrm{mg} / \mathrm{kg}$ or its vehicle administered every other day (days $0,2,4,6$ and 8). Hind paw mechanical sensitivity was assessed with von Frey filaments $2 \mathrm{~h}$ after nitroglycerin administration (blue lines), before nitroglycerin injections and after the end of the treatments on days 10, 12, 14, 16, 18 and 20 (black lines). Tissue samples were obtained at the end. B, C Left panels. Nitroglycerin (red) induces similar acute 
hypersensitivity in males and females, whereas the vehicle (black, $5 \%$ dextrose, $0.105 \%$ propylene glycol) did not induce nociceptive sensitization. B, C Right panels. Chronic administration of nitroglycerin induces a long-lasting hypersensitivity that is persistent exclusively in females, whereas female or male mice treated with vehicle show normal sensitivity. Data are mean \pm S.E.M. ${ }^{* *} p<0.01,{ }^{\star \star *} p<0.001$ vs vehicle. $\# p<0.05, \# \# p<0.01, \# \# \# p<0.001$ vs baseline. 2-way RM ANOVA $n=6$ per condition. NTG, nitroglycerin. Veh, Vehicle.
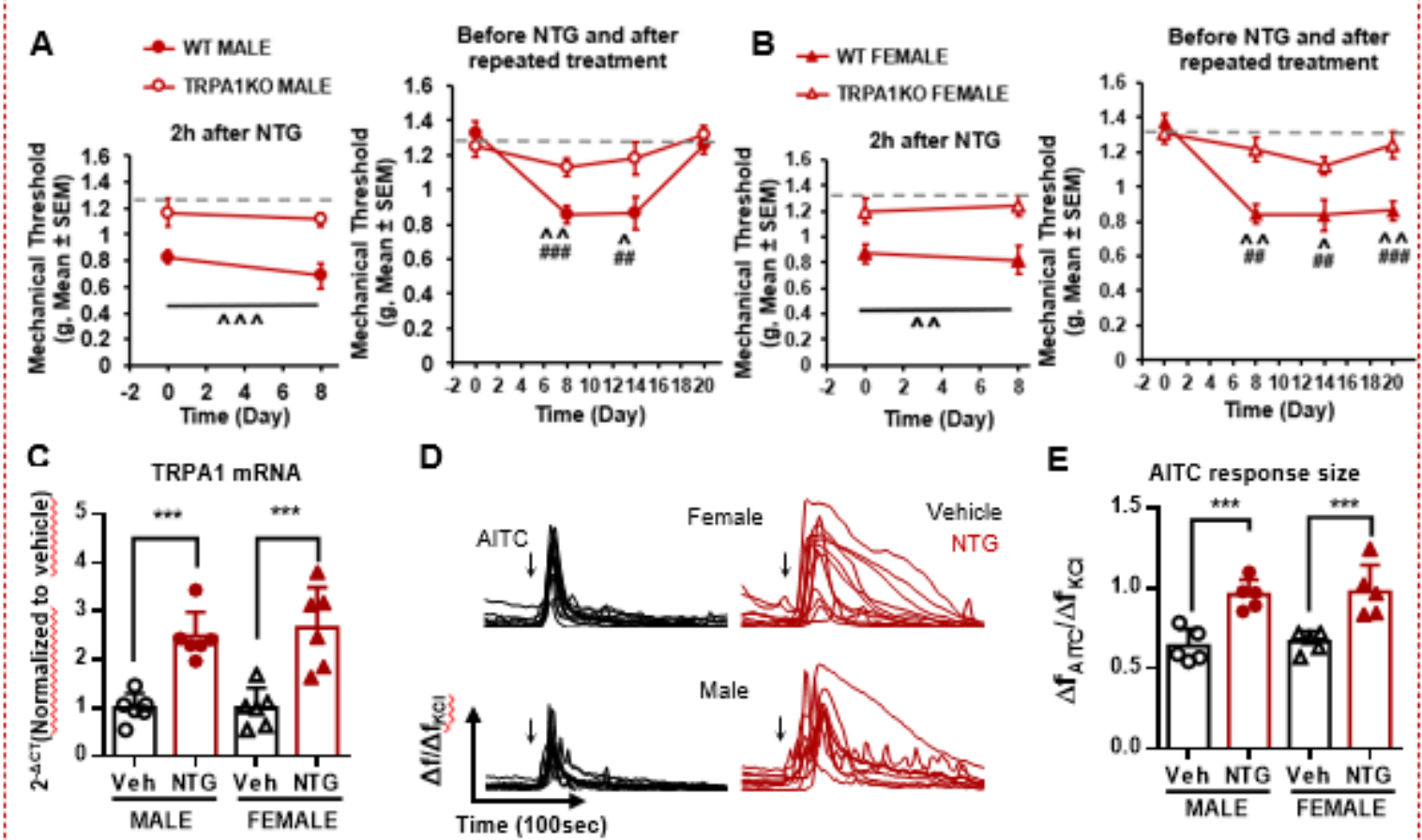

D

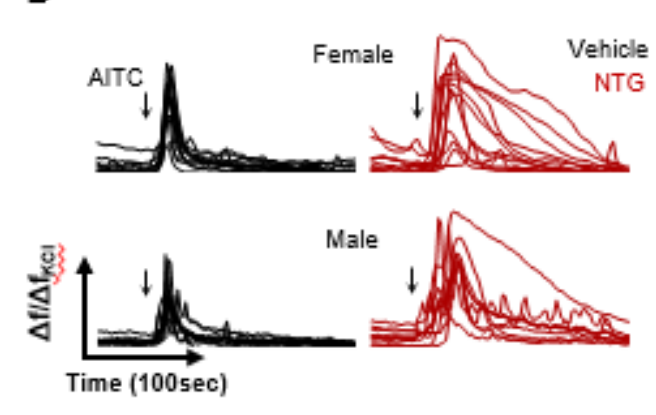

E AITC response size
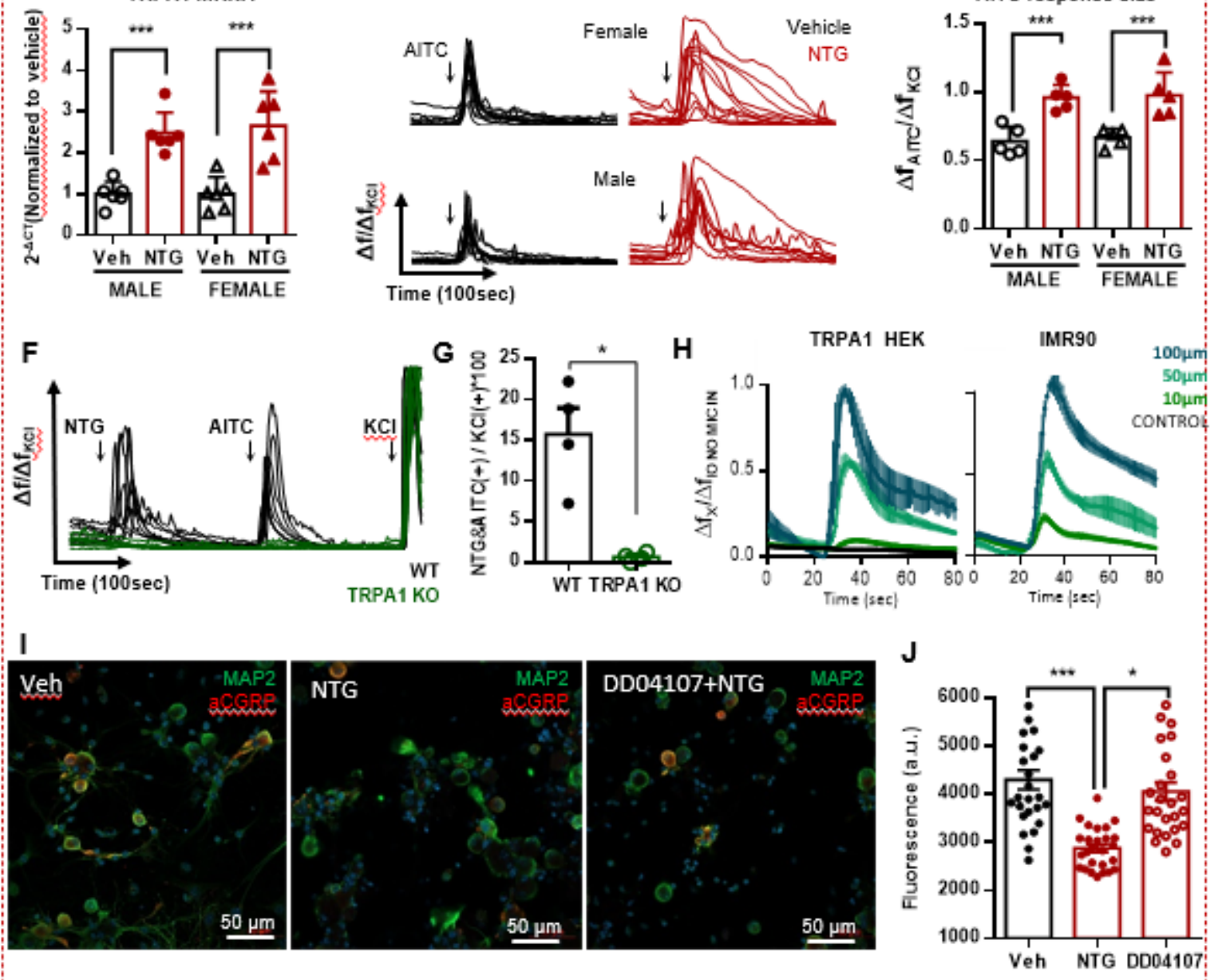

Figure 2

TRPA1-related activity is essential for the development of generalized mechanical hypersensitivity after nitroglycerin treatment and TRPA1 expression and function are increased in trigeminal ganglia of males and females chronically exposed to nitroglycerin. A, B Deletion of TRPA1 prevents the development of 
acute and chronic mechanical hypersensitivity after nitroglycerin in male (A) and female (B) mice. C Trigeminal ganglia of male and female mice chronically exposed to nitroglycerin (red) show increased TRPA1 mRNA expression when compared to vehicle-treated mice (black). D Representative calcium traces in response to AITC $70 \mu \mathrm{M}$ normalized to $\mathrm{KCl} 40 \mathrm{mM}$ in trigeminal ganglia of mice chronically exposed to vehicle or nitroglycerin. E AITC responses are increased in cultures from nitroglycerin-treated animals regardless of the sex. F Neurons responding to nitroglycerin $100 \mu \mathrm{M}$ are also responsive to AITC $70 \mu \mathrm{M}$. These responses are abolished in trigeminal cultures from TRPA1 knockout mice. G $15 \%$ of trigeminal neurons are sensitive to both stimuli in wild-type mice whereas only $1-2 \%$ are responsive in TRPA1 knockout mice. H Human HEK293 cells transfected with TRPA1 gene and IMR90 cells constitutively expressing hTRPA1 show calcium responses after 50, 100 and $500 \mu \mathrm{M}$ nitroglycerin perfusion. I Trigeminal culture exposed to vehicle, nitroglycerin or the exocytosis inhibitor DD04107. Neurons labelled with pan-neuronal marker antiMAP2 in green, antiCGRP in red and nuclei marked blue with DAPI. J CGRP immunoreactivity decreases in cells exposed to nitroglycerin for $30 \mathrm{~min}$ and this decrease is prevented DD04107. Mean mechanical thresholds (A, B) expression (C), calcium fluorescence response $(E \& G)$ or red fluorescence $(J) \pm S$.E.M. Datapoints represent independent experiments. (A \& B) ${ }^{\wedge} \mathrm{p} 0.05,{ }^{\wedge \wedge} \mathrm{p} 0.01,{ }^{\wedge \wedge \wedge} \mathrm{p} 0.001$ vs vehicle. $\# \mathrm{p}<0.05, \# \# \mathrm{p}<0.01$ vs baseline, 2 -way RM ANOVA, $\mathrm{n}=6$ per condition (C, E) ${ }^{* \star *} p<0.001$ 2-way ANOVA, $n=5-6 ;(G) * * p<0.01$ Mann-Whitney U $n=5 ;(J) * p<0.05$, ${ }^{* \star *} p<0.001$, one-way ANOVA. NTG, nitroglycerin. Veh, Vehicle. 


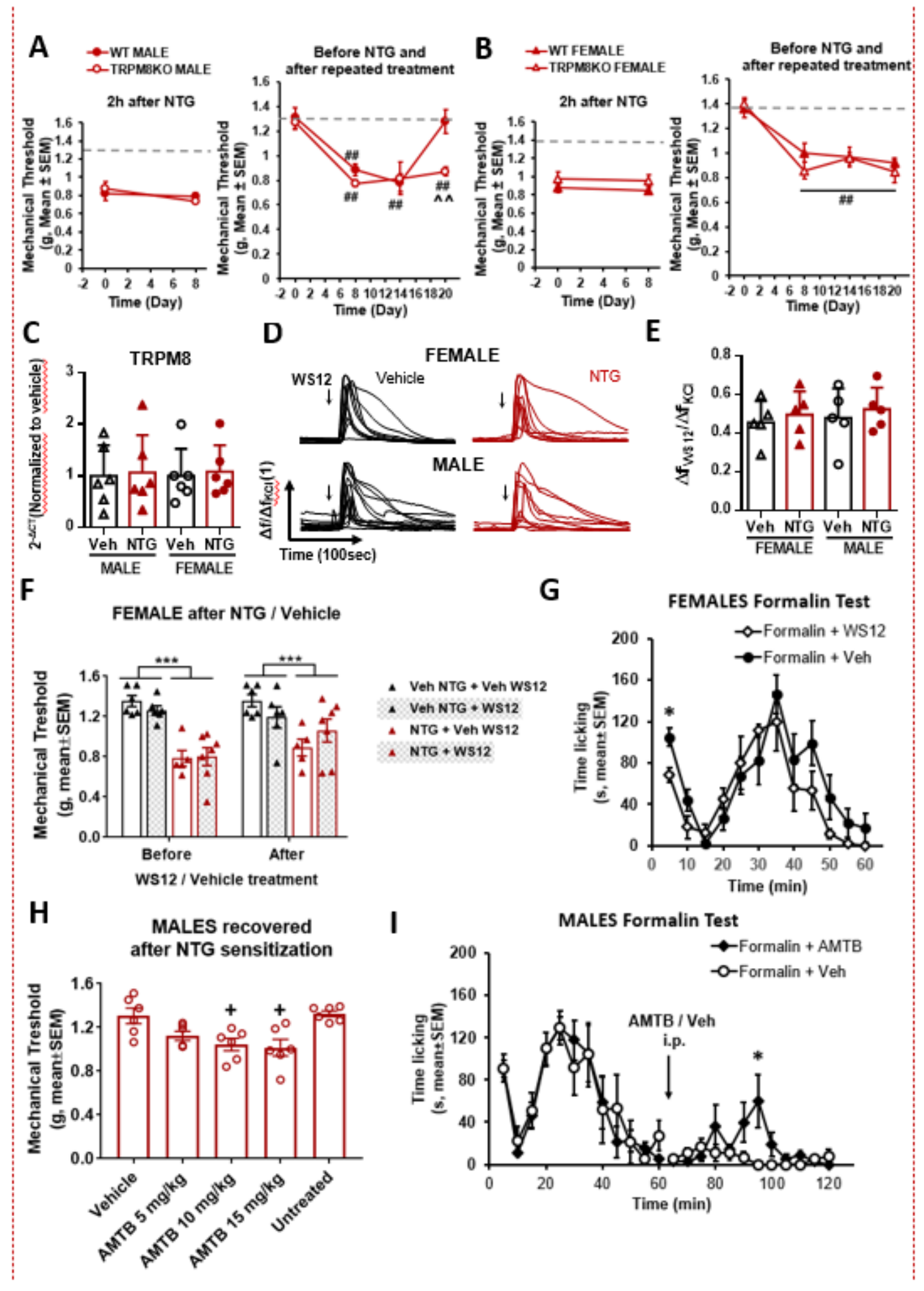

Figure 3

TRPM8 allows recovery of normal sensitivity in male mice exposed to nitroglycerin or formalin. A Wildtype and TRPM8 knockout males display similar acute mechanical hypersensitivity after nitroglycerin treatment (left panel), but the lack of this receptor in prevents the recovery of baseline mechanical sensitivity after the chronic nitroglycerin treatment (right panel) B Acute and long-lasting mechanical hypersensitivity after nitroglycerin treatment is similar in wild-type and TRPM8 knockout female mice. C 
TRPM8 expression in trigeminal ganglia of mice chronically exposed to nitroglycerin or vehicle is similar regardless of the sex or the treatment. D Calcium responses of trigeminal cultures from males and females chronically exposed to nitroglycerin or vehicle when challenged with the selective TRPM8 agonist WS12 (500 nM). E The size of calcium transient currents in response to WS12 is similar in neurons from males and females chronically treated with nitroglycerin or vehicle. F Female mice chronically exposed to nitroglycerin show increased mechanical sensitivity when compared to vehicletreated mice. This sensitivity could not be significantly alleviated after treatment with WS12 $10 \mathrm{mg} / \mathrm{kg}$ i.p. $\mathrm{G}$ Nocifensive behavior in the acute phase of the formalin test $(5 \mathrm{~min})$ is significantly alleviated when females receive formalin co-injected with $6 \mathrm{nmol}$ WS12. H Administration of specific TRPM8 antagonist AMTB (i.p.) to mice chronically exposed to nitroglycerin that already recovered their basal sensitivity unmasks a latent mechanical pain sensitization. I) AMTB $10 \mathrm{mg} / \mathrm{kg}$ administered $1 \mathrm{~h}$ after formalin injections induces significant reinstatement of licking behavior in male mice. Mean mechanical thresholds $(A, B, F \& H)$, expression (C), response size $(E)$, nocifensive behavior $(G, I) \pm S$.E.M. Datapoints of cellular data represent independent experiments. $(A, B) \# \# p<0.01$ vs baseline. ${ }^{\wedge} p 0.01$ vs vehicle 2-way RM ANOVA $n=6$. (C, E) 2-way ANOVA $n=6 / 5$. (F) ${ }^{\star \star *} p<0.001$ nitroglycerin vs vehicle, 3-way ANOVA $n=6-7$. $(G){ }^{*}<0.05$ vs vehicle. Multiple t-test $n=5-6$. $(H)+p<0.05$ vs vehicle Friedman Test $n=6$. $(I) *<0.05$ vs vehicle. Multiple T-test $n=6$ per condition. NTG, nitroglycerin. Veh, Vehicle. 


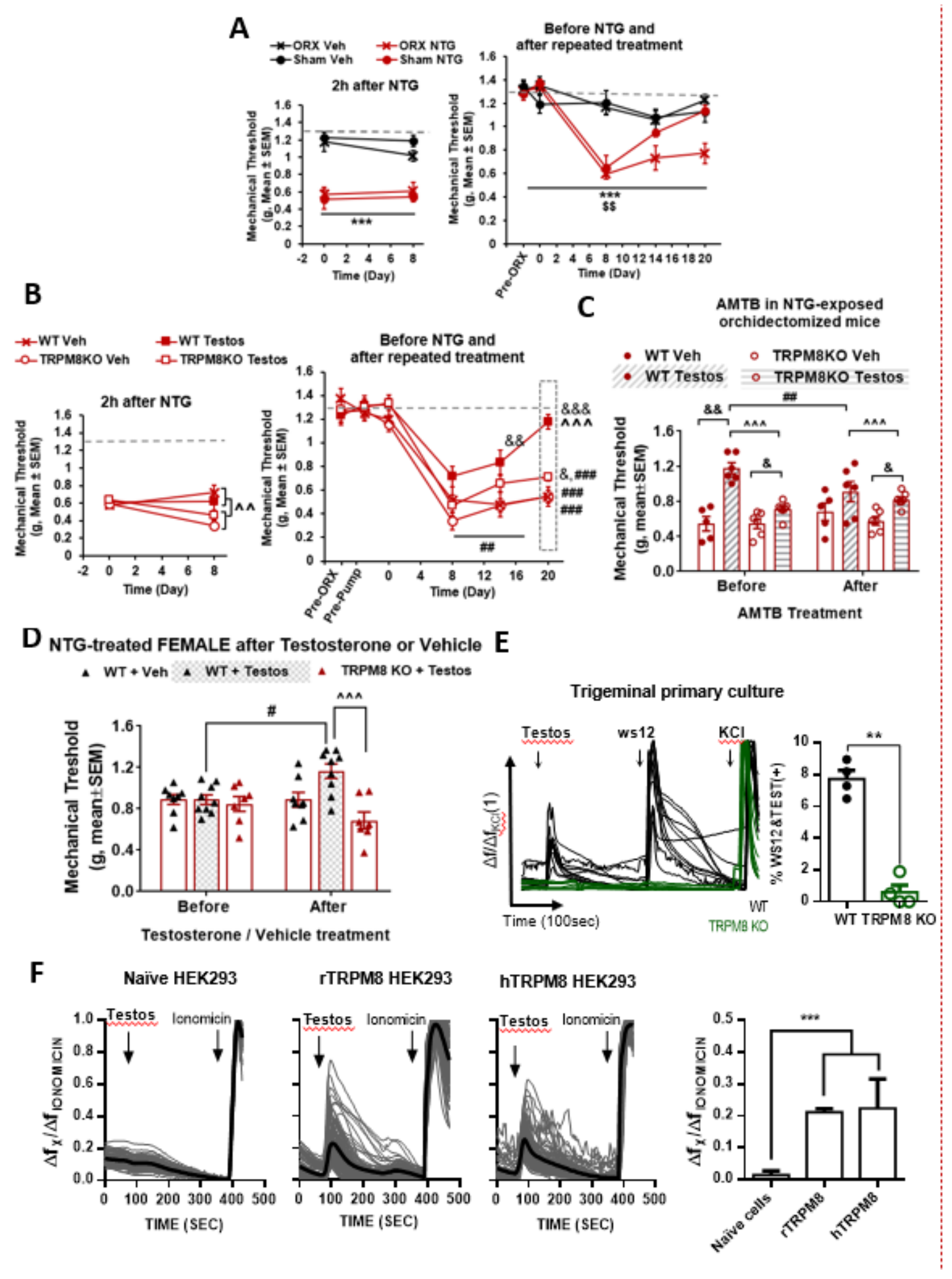

Figure 4

The natural TRPM8 agonist testosterone activates neuronal TRPM8 to promote the recovery of normal mechanosensitivity after the chronic nitroglycerin treatment. A Orchidectomized animals treated with nitroglycerin present similar acute hypersensitivity after nitroglycerin when compared to sham animals (left panel), however orchiectomized mice develop a persistent mechanical hypersensitivity (right panel). B Left panel, orchiectomized animals receiving testosterone supplementation or its vehicle show similar 
acute hypersensitivity after the first dose of nitroglycerin, however orchidectomized TRPM8 knockout mice develop stronger sensitization after the last nitroglycerin dose, regardless of the hormonal treatment. Right panel, mice receiving testosterone supplementation show inhibition of the long-lasting hypersensitivity induced after chronic nitroglycerin treatment. This attenuation leads to recovery of normal sensitivity in wild-type mice, but not in TRPM8 knockout mice. C Testosterone-supplemented wildtype mice that recovered their normal sensitivity reinstate their pain sensitization after administration of TRPM8 blocker AMTB on day 21. This response is absent in control and TRPM8 knockout mice. D Female mice suffering from persistent hypersensitivity presented rapid recovery of basal sensitivity values $1 \mathrm{~h}$ after i.p. testosterone $1 \mathrm{mg} / \mathrm{kg}$. E Cultured trigeminal neurons showing transient calcium currents after testosterone $10 \mathrm{nM}$ are also sensitive to WS12 (500 nM) and cultured trigeminal neurons of TRPM8 knockout mice are largely unresponsive to both stimuli. Right graph, 7\% of neurons from trigeminal cultures of wild-type mice show transient currents after application of testosterone $10 \mu \mathrm{M}$, while this response is abolished in TRPM8 knockout mice. F Testosterone 10 pM activates HEK293 cells expressing murine and human TRPM8 while this response is absent in control cells. Mean mechanical thresholds $(A, B, C, D)$, response size $(E, F) \pm$ S.E.M. Datapoints of cell culture experiments represent independent experiments. $(A) * * * p<0.001$ vs nitroglycerin vehicle, $\$ \$ p<0.01$ vs sham, 3-way RM ANOVA. (B) ${ }^{\wedge \wedge} p<0.01,{ }^{\wedge \wedge} p<0.001$ vs TRPM8 KO; \&p<0.05, \&\&p<0.01 vs testosterone vehicle; \#\#p<0.01, \#\#\#p<0.001 vs. baseline, 3-way RM ANOVA $n=6$. (C) ${ }^{\wedge \wedge} p<0.001$ vs TRPM8 KO; $\& p<0.05$, vs testosterone vehicle; \#\#p<0.01 vs. basal, 3-way RM ANOVA $n=6$. (D) ${ }^{\wedge \wedge}{ }^{\wedge} p<0.001$ vs TRPM8 KO, \#p<0.05 vs. basal, 2way ANOVA $n=7-9$. (E) * $p<0.05$ Mann Whitney $U n=4$. (F) ${ }^{* \star *} p<0.001$ vs control, Mann-Whitney $n>80$ per group. NTG, nitroglycerin. Veh, Vehicle. Testos, testosterone. 
A

WS12 - rTRPM8

$\Delta \mathrm{G}(\mathrm{kcal} / \mathrm{mol})=9.04 ; \mathrm{Kd}(\mathrm{nM})=234.5$

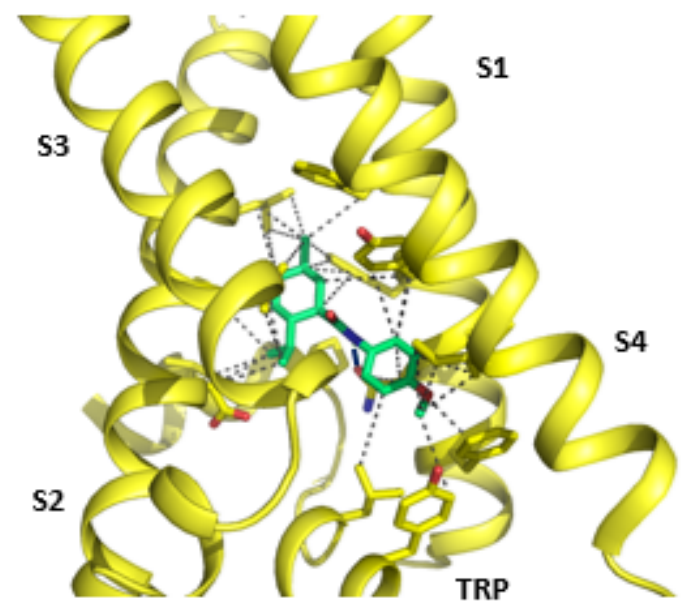

C

Progesterone - rTRPM8

$\Delta \mathrm{G}(\mathrm{kcal} / \mathrm{mol})=8.16 ; \mathrm{Kd}(\mathrm{nM})=1036.7$
B

Testosterone - rTRPM8

$\Delta \mathrm{G}(\mathrm{kcal} / \mathrm{mol})=9.25 ; \mathrm{Kd}(\mathrm{nM})=164.5$

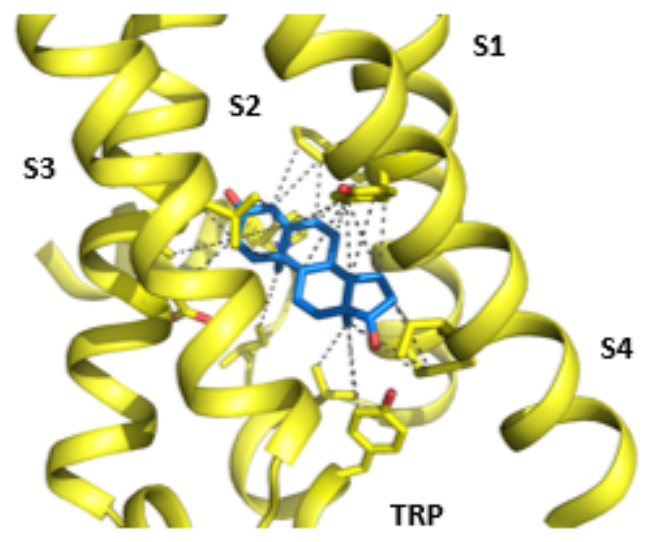

D

Estradiol - rTRPM8

$\Delta \mathrm{G}(\mathrm{kcal} / \mathrm{mol})=8.97 ; \mathrm{Kd}(\mathrm{nM})=264$
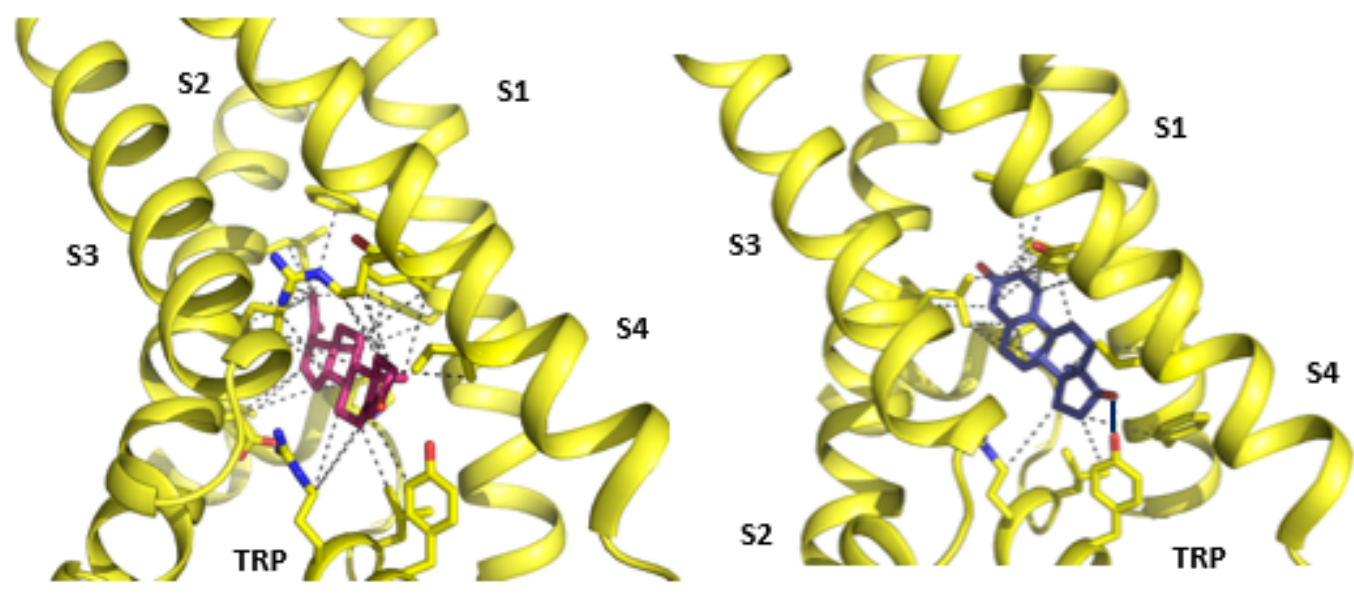

Figure 5

Binding of the menthol derivative WS12 and sexual hormones to the rat TRPM8 menthol pocket after local docking simulations. A WS12 B Testosterone C Progesterone D Estradiol. The TRPM8 structure is shown as yellow cartoon representing with sticks the side chains involved in the binding. WS12 and testosterone are shown as sticks of different color. Gray-dashed lines indicate Van der Waals interactions with ligands, whereas blue-solid lines are hydrogen bonds. Hydrogen atoms have been removed. S1, S2, S3, S4 and TRP are TRPM8 transmembrane domains. $\Delta \mathrm{G}$ is an estimation of the binding energy. $\mathrm{Kd}$ is the dissociation constant calculated with $\Delta \mathrm{G}$ using the equation $\Delta \mathrm{G}=\mathrm{R} \cdot \mathrm{T} \cdot \operatorname{InKd}$. 


\section{Supplementary Files}

This is a list of supplementary files associated with this preprint. Click to download.

- SuppIMaterialStatisticalDataok.docx

- SupplementaryFigure1.pptx

- SupplementaryFigure2.pptx

- SupplementaryFigure3.pptx

- SupplementaryFigure4.pptx

- SupplementaryFigure5.pptx 